\title{
First Look at Average-Case Complexity for Planar Maximum-Likelihood Detection ${ }^{\S}$
}

\author{
Kai-Kit Wong \\ Centre for Communications Systems and Technology \\ The University of Hull \\ Kingston Upon Hull, HU6 7RX \\ United Kingdom \\ Email: k.wong@hull .ac.uk
}

\author{
Nikos Mamoulis \\ Department of Computer Science \\ The University of Hong Kong \\ Pokfulam Road \\ Hong Kong \\ Email: nikos@Cs.hku.hk
}

\begin{abstract}
In this paper, an efficient exact maximum-likelihood (ML) detection scheme is presented for a multiple-input singleoutput (MISO) system with real signal constellations. The proposed technique has a geometrical interpretation of exploring the points jointly "close" in all coordinate axes around the decoding hyperplane and is therefore dubbed planar detection. The fact that the lattice points which are close in all coordinate axes are much less, leads to dramatic reduction in detection complexity. Making a few approximations, this paper derives the averagecase complexity exponent, $e_{c}$, for planar detection analytically in a closed form. Numerical results show that for an $(n, 1)^{1}$ system, although the expected complexity is still exponential, complexity reduction of 2 exponents, i.e., from $e_{c}$ to $e_{c}-2$, is realized and such advantage is promised irrespective of the size of the signal constellations and the received signal-to-noise ratio (SNR).
\end{abstract}

\section{INTRODUCTION}

Maximum-likelihood (ML) detection is well understood to be the optimal detection strategy for both multiuser detection [1]-[3] and space-time decoding (e.g., [4]). The promising performance of ML detection, however, comes with the challenge of exponential complexity increase.

For an $\left(N_{t}, N_{r}\right)$ system where $N_{t} \leq N_{r}$ (i.e., an overdetermined system), ML detection can be achieved in a relatively cheap way by sphere decoding [5]-[9]. In sphere decoding, the channel is rotated onto a space in a way that the spatially multiplexed signals are only sequentially dependent. An efficient algorithm is hence available to search the signal points that fall inside the decoding hypersphere for a given radius. As a result, the ML detection can then be efficiently obtained by solving the dual spherical search problem with a judicious choice of radius. Further reduction in decoding complexity can be obtained by suboptimal detection techniques such as zeroforcing, nulling and cancellation [10], which albeit degrade the diversity performance severely.

In practice, however, it is unlikely to have sufficient number of receive antennas for decoupling the spatial signals (which may include the interfering signals). It is thus more reasonable to consider the case where $N_{t}>N_{r}$ (i.e., an underdetermined

\footnotetext{
$\S$ This work was supported in part by The Hong Kong Research Grants Council under grant HKU7 142/04E.

${ }^{1}$ The notation $\left(N_{t}, N_{r}\right)$ is used to denote a multiple-input multiple-output (MIMO) system which has $N_{t}$ transmitting inputs and $N_{r}$ receiving outputs.
}

system). Unfortunately, the immediate challenge is that none of the existing efficient detectors (both optimal and suboptimal ones) would work.

This paper investigates the complexity reduction for the ML detection problem of a multiple-input single-output (MISO) system, i.e., $(n, 1)$, which forms the basis of asymmetric fat MIMO-ML detection (or $n=N_{t}-N_{r}+1$ ). In particular, we shall present a detection scheme, which assures to give exactML detection at reduced complexity for $(n, 1)$ systems if the signal constellation is real, e.g., pulse-amplitude modulation (PAM).

Our approach is based on the geometrical understanding that the ML lattice point appears to be a point that is the first seeing the hyperplane (so-called "close") in all coordinate axes. And, given the fact that these close points are scarce, an efficient algorithm exists to greatly reduce the detection complexity. In light of this interpretation, the proposed detection scheme is therefore called planar detection [11].

In contrast to the previous work [11], the main contribution of this paper lies in the analytical derivation of the averagecase complexity exponent, $e_{c} \triangleq \frac{\log \mathcal{C}(n, \mathrm{SNR})}{\log n}$, in a closed form for planar detection where $\mathcal{C}(n, S N R)$ denotes the expected computational complexity in the number of elementary calculations over many independent channel instantiations and transmit lattices. The complexity exponent derived serves as a complexity measure for ideal realization of planar detection and can provide a reasonable estimate on the average complexity for actual implementation of planar detection.

The remainder of the paper is organized as follows. Section II will describe the channel model for real-valued MISOML detection. In Section III, we present the so-called planar detection for efficient exact MISO-ML detection for real signal constellations. Section IV is dedicated for the derivation of the average-case complexity. Simulation results are provided in Section V, and we conclude the paper in Section VI.

\section{MISO-ML DETECTION}

For a spatially multiplexed MISO system, each transmitter or antenna is sending an independent symbol, $x_{k} \in \mathcal{Q}$, which takes values from the symbol set $\mathcal{Q}$. At the receiver side, the 
received signal can be written as

$$
y=h_{1} x_{1}+h_{2} x_{2}+\cdots+h_{n} x_{n}+\eta
$$

where $h_{k}$ is the channel response from the $k$ th transmitter to the receiver, and $\eta$ denotes the noise, which has zero-mean Gaussian distribution with variance of $\sigma_{n}^{2}$.

To simplify our discussion, we shall assume that all the variables are real-valued. It is however possible to generalize the results in this paper for complex values (for details, see [11]). ML detection aims to find the values, $x_{1}, x_{2}, \ldots, x_{n}$, jointly or the vector $\mathbf{x}=\left[\begin{array}{llll}x_{1} & x_{2} & \ldots & x_{n}\end{array}\right]^{T}$ (the superscript $T$ denotes transposition) that

$$
\mathbf{x}_{\mathrm{ML}}=\arg \min _{\mathbf{x} \in \mathcal{Q}^{n}}\left(h_{1} x_{1}+h_{2} x_{2}+\cdots+h_{n} x_{n}-y\right)^{2} .
$$

Geometrically, it requires to find the point $\left(x_{1}, x_{2}, \ldots, x_{n}\right) \in$ $\mathcal{Q}^{n}$ that is the closest to the decoding hyperplane (see Figure 1 for $\mathcal{Q}=\{-3,-1,1,3\}$ and $n=2$ )

$$
\mathcal{P}: \quad h_{1} x_{1}+h_{2} x_{2}+\cdots+h_{n} x_{n}=y, \quad \mathbf{x} \in \mathcal{R}^{n}
$$

\section{Planar DETECTION}

Before we describe the algorithm that can efficiently solve the ML detection (2), we find it useful to define the closeness of a point.

Definition III-1 Closeness of a point-Assuming for the sake of simplicity that the channels are real and positive, i.e., $h_{1}, h_{2}, \ldots \geq 0$, a point $\left(x_{1}, x_{2}, \ldots, x_{n}\right)$ is said to be close in coordinate axis or direction $k$ (or abbreviated as d- $k$ ) if $x_{k}$ is the first point that sees the decoding hyperplane from the half-space $h_{1} x_{1}+\cdots+h_{n} x_{n}>y$ or $h_{1} x_{1}+\cdots+h_{n} x_{n}<y$, i.e.,

$$
\begin{aligned}
x_{k} \in\left\{x: \min _{x \in \mathcal{Q}} x \text { s.t. } x>\frac{y-\sum_{m \neq k} h_{m} x_{m}}{h_{k}}\right\} \\
\cup\left\{x: \max _{x \in \mathcal{Q}} x \text { s.t. } x<\frac{y-\sum_{m \neq k} h_{m} x_{m}}{h_{k}}\right\} .
\end{aligned}
$$

It should be noted that the closeness property of a point in one particular direction is in fact conditioning on the coordinates in other directions. In other words, if some coordinates change, the closeness property may be destroyed.

Theorem III-1 Joint closeness of the ML lattice pointThe point $\left(x_{1}, x_{2}, \ldots, x_{n}\right) \in \mathcal{Q}^{n}$ that corresponds to the ML detection must be close in all directions (or close in d$1,2, \ldots, n)$.

Proof: See [11].

The joint closeness property serves as a necessary condition for ML detection. The fact that the number of points that are jointly close in all directions are scarce becomes the key for an efficient detection algorithm to be found. In Figure 1, we show an example of a decoding line given $\mathcal{Q}=\{-3,-1,1,3\}$. In this example, there are six lattice points which are close in d-1,2. As compared to the total of 16 possible lattice points, if we search only the closed points, it can significantly reduce the decoding complexity while still achieving the exact $\mathrm{ML}$ performance. In the following, we give a formal description of our Planar Detection or Plane Decoding algorithm:

1) Initialize $k=1$. Then, randomly pick a point $\mathbf{x}^{(1)}=$ $\left[x_{1}^{(1)} x_{2}^{(1)} \ldots\right]^{T} \in \mathcal{Q}^{n}$ and define a set $\mathbf{X} \triangleq\left\{\mathbf{x}^{(1)}\right\}$. Compute

$$
\triangle y\left(\mathbf{x}^{(1)}\right)=h_{1} x_{1}^{(1)}+h_{2} x_{2}^{(1)}+\cdots+h_{n} x_{n}^{(1)}-y .
$$

2) If $\mathbf{X}$ is empty, then go to Step 6. Otherwise, find the two coordinates that make $\mathbf{x}^{(1)}$ close in $\mathrm{d}-k$

$$
\hat{x}_{k}=\left\{x: \arg \min _{x \in \mathcal{Q}} x \quad \text { s.t. } x>x_{\mathrm{B}}\right\}
$$

and

$$
\check{x}_{k}=\left\{x: \arg \max _{x \in \mathcal{Q}} x \text { s.t. } x<x_{\mathrm{B}}\right\}
$$

where

$$
x_{\mathrm{B}}=x_{k}^{(1)}-\frac{\triangle y\left(\mathbf{x}^{(1)}\right)}{h_{k}} .
$$

For each of the new coordinates if not empty, produce a new point $\hat{\mathbf{x}}$ and compute

$$
\triangle y(\hat{\mathbf{x}})=h_{k}\left(\hat{x}_{k}-x_{\mathrm{B}}\right)
$$

Similarly, we shall have also $\breve{\mathrm{x}}$ and $\triangle y(\breve{\mathbf{x}})$.

3) If $\hat{x}_{k}=x_{k}^{(1)}$, the point $\mathbf{x}^{(1)}$ is close in $\mathrm{d}-1,2, \ldots, k$. Then do the followings: if $k<n$, update $k:=k+1$ and $\mathbf{X}:=[\mathbf{X} \check{\mathbf{x}}]$. Otherwise, if $k=n$, erase $\mathbf{X}^{(1)}$ from $\mathbf{X}$ and reset $k=1$. The point $\mathbf{X}^{(1)}$ is a point jointly close in all directions and is thus a candidate for ML detection and is stored separately. Go back to Step 2 .

4) If $\check{x}_{k}=x_{k}^{(1)}$, the point $\mathbf{x}^{(1)}$ is close in $\mathrm{d}-1,2, \ldots, k$. Then do the followings: if $k<n$, update $k:=k+1$ and $\mathbf{X}:=[\mathbf{X} \hat{\mathbf{X}}]$. Otherwise, if $k=n$, erase $\mathbf{X}^{(1)}$ from $\mathbf{X}$ and reset $k=1$. The point $\mathbf{X}^{(1)}$ is a point jointly close in all directions and is thus a candidate for $\mathrm{ML}$ detection and is stored separately. Go back to Step 2 .

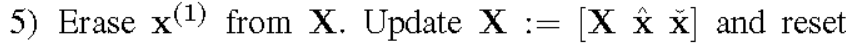
$k=1$. Go back to Step 2 .

6) All of the points jointly close in all directions are found. Among these points, the one that gives the least $\triangle y^{2}(\mathrm{x})$ is the ML detection point.

In the above algorithm, we have assumed that $h_{1}, h_{2}, \ldots \geq$ 0 . However, if some channels are negative, one can still apply the above algorithm for the channels, $\left|h_{1}\right|,\left|h_{2}\right|, \ldots,\left|h_{n}\right|$. But the ML solution has to be modified by flipping the signs of the symbols for the negative channels.

Figure 1 illustrates an example showing the decoding procedure of planar detection. As can be seen, eight points are actually visited instead of six points. The reason is that some addiional points will need to be visited to navigate along the decoding hyperplane for the search of the jointly close points. 


\section{AVERAge-CAse CompleXity}

To gain understanding on the average complexity involved, this section is devoted to give the first look at the expected computational complexity of planar detection averaged over independent fading channels and the transmit sequences $\mathbf{x} \in$ $\mathcal{Q}^{n}$ assuming ideal implementation, i.e., all the visited points during detection are jointly close or overheads are ignored.

$|\mathcal{Q}|$-PAM with signal constellations as shown in Figure 2 is considered so that

$$
\mathcal{Q}=\{ \pm 0.5 d, \pm 1.5 d, \ldots, \pm 0.5(|\mathcal{Q}|-1) d\}
$$

where $d$ is the separation of every adjacent constellations and $|\mathcal{Q}|$ denotes the number of constellation points. As a result, the average transmit energy per dimension, $\sigma_{x}^{2} \triangleq \mathrm{E}\left[x_{k}^{2}\right]$, is given by

$$
\sigma_{x}^{2}=\frac{2}{|\mathcal{Q}|} \sum_{\ell=1}^{|\mathcal{Q}| / 2}\left(\ell-\frac{1}{2}\right)^{2} d^{2}
$$

Denoting $d_{\mathrm{c}}=(|\mathcal{Q}|-1) d$ as the distance between the two endpoints of $\mathcal{Q}$ and then simplifying (11), we have

$$
\sigma_{x}^{2}=\frac{d_{\mathrm{c}}^{2}}{12}\left(\frac{|\mathcal{Q}|+1}{|\mathcal{Q}|-1}\right)
$$

Note that if $x_{k} \in \mathcal{Q}$ (defined in (10)), $\mathbf{x} \in \mathcal{Q}^{n}$ will form the lattice space of a hypercube. Nevertheless, for simplicity sake, we shall approximate it by the lattice space of a hypersphere and this approximation greatly simplifies the calculation of the intersection between the decoding hyperplane and the lattice space later on. To preserve the same transmit energy, we set

$$
d_{\mathrm{c}}^{n}=\frac{\pi^{\frac{n}{2}}}{\mathcal{I}(n)} R^{n}
$$

where

$$
\mathcal{I}(n)= \begin{cases}\left(\frac{n}{2}\right) ! & \text { if } n \text { is even, } \\ \frac{\sqrt{\pi}}{2^{n}} \frac{n !}{\left(\frac{n-1}{2}\right) !} & \text { if } n \text { is odd, }\end{cases}
$$

and $R$ denotes the radius of the lattice hypersphere

$$
\mathcal{S}: x_{1}^{2}+x_{2}^{2}+\cdots+x_{n}^{2} \leq R^{2}, \quad \mathbf{x} \in \mathcal{Q}^{n} .
$$

For ideal implementation of planar detection, the averagecase complexity allows the following expansion:

$$
\mathcal{C}(n, \mathrm{SNR}) \approx 4 \mathrm{E}\left[\mathcal{N}_{\text {close }}\right]
$$

where $\mathcal{N}_{\text {close }}$ denotes the number of lattice points jointly close in all directions and we have used the fact that only four elementary computations are required for each visited point (see (8) and (9)). To know the complexity $\mathcal{C}$, it requires the estimation of the average number of $\mathcal{N}_{\text {close }}$.

For a given channel state $\left(h_{1}, h_{2}, \ldots, h_{n}\right)$ and the transmit lattice $\mathbf{x}$, the number of jointly close points, $\mathcal{N}_{\text {cose }}$, depends greatly on the intersectional area (or volume if $n>3$ ) between the lattice space and the decoding hyperplane. This can be exemplified in Figure 3 where the number of jointly close points is shown for the decoding line $2 x_{1}+3 x_{2}=y$ with various $y$. As can be seen, $\mathcal{N}_{\text {close }}$ is, by and large, proportional to the sectional length between the decoding line and the lattice space. Also, it should be noted that $\mathcal{N}_{\text {close }}$ depends largely on $y$ (i.e., the shortest distance of the decoding line from the origin) but not the channel state. Therefore, for $n=2$, we have

$$
\mathcal{N}_{\text {close }} \approx\left(\frac{N_{\max }-1}{\mathcal{L}_{\max }}\right) \mathcal{L}(y)+1
$$

where $\mathcal{L}(y)$ denotes the sectional length as a function of $y$ and $N_{\max }$ denotes the maximal possible number of jointly close points. (17) is written in a form such that when $\mathcal{L}(y)=0$, then $\mathcal{N}_{\text {close }}=1$ while if $\mathcal{L}(y)=\mathcal{L}_{\text {max }}$, then $\mathcal{N}_{\text {close }}=N_{\text {max }}$. In this example, $N_{\max }=6$ which occurs when the decoding line intersects with the stepwise border lines shown in Figure 3. For general $|\mathcal{Q}|$-PAM, it can be easily seen that $N_{\max } \approx 2|\mathcal{Q}|$ if $n=2$.

This idea is illustrated in Figure 4 and is generalized here for $n>2$ so that

$$
\mathcal{N}_{\text {close }}=\left(\frac{N_{\max }-1}{\mathcal{V}_{\max }}\right) \mathcal{V}(y)+1
$$

where $\mathcal{V}$ denotes the intersectional area or volume. Because the intersection of the decoding hyperplane $\mathcal{P}$ and the lattice hypersphere $\mathcal{S}$ is another hypersphere, the intersectional volume can be found as

$$
\mathcal{V}(y)=\left\{\begin{array}{cl}
\frac{\pi^{\frac{n-1}{2}}}{\mathcal{I}(n-1)}\left(R^{2}-y^{2}\right)^{\frac{n-1}{2}} & \text { if }|y| \leq R \\
0 & \text { if }|y|>R
\end{array}\right.
$$

Moreover, we know that $\mathcal{V}_{\max }=\mathcal{V}(0)$ and $N_{\max } \approx 2|\mathcal{Q}|^{n-1}$. (18) can therefore be expressed as

$\mathcal{N}_{\text {dose }}=\left\{\begin{array}{cl}\left(2|\mathcal{Q}|^{n-1}-1\right)\left[1-\left(\frac{y}{R}\right)^{2}\right]^{\frac{n-1}{2}}+1 & \text { if }|y| \leq R \\ 1 & \text { if }|y|>R\end{array}\right.$

To find the expected value of $\mathcal{N}_{\text {close }}$, we need the probability distribution of $y$. Recognizing that

$$
y=h_{1} x_{1}^{(t)}+h_{2} x_{2}^{(t)}+\cdots+h_{n} x_{n}^{(t)}+\eta
$$

where $\left(x_{1}^{(t)}, x_{2}^{(t)}, \ldots, x_{n}^{(t)}\right)$ is the actual lattice point being transmitted, a random model for the channel and the transmit lattice is required. In this paper, we assume that $h_{k}$ 's are independent identically distributed (i.i.d.) zero-mean unit-variance Gaussian random variables, i.e., $\mathrm{E}\left[h_{k}\right]=0$ but $\mathrm{E}\left[h_{k}^{2}\right]=1 \quad \forall k$. Likewise, $x_{k}^{(t)}$ 's are i.i.d. uniform distributed discrete random variables from the set $\mathcal{Q}$. If $n$ is large, e.g., $n>3$, by the central limit theorem, $y$ will be nearly Gaussian with

$$
\mathrm{E}[y]=0
$$

and

$$
\mathrm{E}\left[y^{2}\right]=n \sigma_{x}^{2}+\sigma_{n}^{2}
$$

so that

$$
f_{Y}(y)=\frac{1}{\sqrt{2 \pi\left(n \sigma_{x}^{2}+\sigma_{n}^{2}\right)}} e^{-\frac{y^{2}}{2\left(n \sigma_{x}^{2}+\sigma_{n}^{2}\right)}}
$$




$$
\begin{aligned}
\mathrm{E}\left[\mathcal{N}_{\text {close }}\right] & =\int_{R}^{\infty}+\int_{-\infty}^{-R} f_{Y}(y) d y+\int_{-R}^{R}\left\{\left(2|\mathcal{Q}|^{n-1}-1\right)\left[1-\left(\frac{y}{R}\right)^{2}\right]^{\frac{n-1}{2}}+1\right\} f_{Y}(y) d y \\
& =1+\frac{2|\mathcal{Q}|^{n-1}-1}{\sqrt{2 \pi}} \int_{-\frac{R}{\sqrt{n \sigma_{x}^{2}+\sigma_{n}^{2}}}}^{\frac{R}{\sqrt{n \sigma_{x}^{2}+\sigma_{n}^{2}}}}\left[1-\left(\frac{n \sigma_{x}^{2}+\sigma_{n}^{2}}{R^{2}}\right) y^{2}\right]^{\frac{n-1}{2}} e^{-\frac{y^{2}}{2}} d y
\end{aligned}
$$

$$
\mathrm{E}\left[\mathcal{N}_{\text {close }}\right]=1+\frac{2|\mathcal{Q}|^{n-1}-1}{\sqrt{2 \pi}}\left(\frac{R}{\sqrt{n \sigma_{x}^{2}+\sigma_{n}^{2}}}\right) \int_{-\frac{\pi}{2}}^{\frac{\pi}{2}} \cos ^{n} \theta e^{-\frac{R^{2}}{2\left(n \sigma_{x}^{2}+\sigma_{n}^{2}\right)} \sin ^{2} \theta} d \theta
$$

As a consequence, we get (25) (see the top of the next page). Using some mathematical manipulations, it can further be simplified as (26). Now, define

$$
\mathrm{SNR} \triangleq \frac{\sum_{k=1}^{n} \mathrm{E}\left[x_{k}^{2}\right]}{\mathrm{E}\left[\eta^{2}\right]}=\frac{n \sigma_{x}^{2}}{\sigma_{n}^{2}}
$$

and note that $R=\mu \sigma_{x}$ where

$$
\mu=\sqrt[n]{\mathcal{I}(n)} \sqrt{\frac{12}{\pi}} \sqrt{\frac{|\mathcal{Q}|-1}{|\mathcal{Q}|+1}}
$$

As such, we have also

$$
\frac{R}{\sqrt{n \sigma_{x}^{2}+\sigma_{n}^{2}}}=\frac{\mu}{\sqrt{n}} \frac{\sqrt{\mathrm{SNR}}}{\sqrt{\mathrm{SNR}+1}}
$$

To summarize, we can now write the average-case complexity $\mathcal{C}(n, \mathrm{SNR})$ as (30) (see the top of the next page) with $\mu$ previously defined in (28). Additionally, the complexity exponent, $e_{c}$, can be readily found as

$$
e_{c}=\frac{\log \mathcal{C}(n, \mathrm{SNR})}{\log n} .
$$

It is often useful to consider $e_{c}$ for complexity comparisons because if $e_{c}$ approaches a constant, then the complexity is polynomial but if $e_{c}$ grows like $\frac{n}{\log n}$, then the complexity is exponential. For brute-force ML detection of a MISO system, $\mathcal{C}(n, \mathrm{SNR})=|\mathcal{Q}|^{n}(2 n+1)$ and the complexity exponent $e_{c}$ will grow like $\frac{n}{\log n}$. Therefore, the complexity is exponential.

\section{Simulation Results}

Computer simulations have been done to evaluate the decoding complexity for various size of signal constellations $|\mathcal{Q}|$ and the number of dimensions $n$. Complexity exponent results for both the planar detection based on the derivation and the brute-force ML detection are provided for comparisons, and are plotted in Figure 5. In this figure, SNR is assumed to be $15 \mathrm{~dB}$, but it should be noted that the results are insensitive to the SNR.

Results demonstrate that the complexity of planar detection is still exponential as it grows linearly with $n$. This is however not surprising because there is no channel structure that can be exploited to simplify the detection (note that in a square MIMO, sphere decoding simplifies the detection by rotating the channel into a triangular structure). Having this in mind, planar detection in fact significantly reduces the complexity as compared to the brute-force ML detection, the only choice for MISO-ML. In particular, planar detection achieves a complexity reduction from $e_{c}$ to $e_{c}-2$ irrespective of the signal constellation size $|\mathcal{Q}|$ and the SNR without compromising the ML performance.

\section{CONCLUSION}

This paper has presented an efficient detection method that achieves exact-ML performance for MISO real signal constellations. The average-case complexity exponent has also been derived in a closed form.

\section{REFERENCES}

[1] T. Van Etten, "Maximum likelihood receiver for multiple channel transmission systems," IEEE Trans. Commun., pp. 276-283, Feb. 1976.

[2] S. Verdú, "Minimum probability of error for asynchronous Gaussian multiple-access channels," IEEE Trans. Info. Theory, vol. 32, no. 1, pp. 85-96, Jan. 1986.

[3] H. Artés, D. Seethaler, and F. Hlawatsch, "Efficient decoding algorithms for MIMO channels: a geometrical approach to approximate ML detection," IEEE Trans. Sig. Proc., 2004.

[4] V. Tarokh, N. Seshadri, and A. R. Calderbank, "Space-time codes for high data rate wireless communication: performance criterion and code construction," IEEE Trans. Info. Theory, vol. 6, pp. 744-765, Mar. 1998.

[5] U. Fincke, and M. Phost, "Improved methods for calculating vectors of short length in a lattice, including a complexity analysis," Math. of Comp., vol. 44, pp. 463-471, Apr. 1985.

[6] O. Damen, A. Chkeif, and J. C. Belfiore, "Lattice code decoder for spacetime codes," IEEE Commun. Letters, vol. 4, no. 5, pp. 161-163, May 2000 .

[7] E. Viterbo, and J. Boutros, "A universal lattice code decoder for fading channels," IEEE Trans. Info. Theory, vol. 45, pp. 1639-1642, Jul. 1999.

[8] A. M. Chan, and I. Lee, "A new reduced-complexity sphere decoder for multiple antenna systems," in Proc. IEEE Int. Conf. Commun., vol. 1, pp. 460-464, 28 Apr.-2 May 2002.

[9] K. K. Wong, and A. Paulraj, "On the decoding order of MIMO maximumlikelihood sphere decoder: linear and non-linear receivers," in Proc. IEEE Veh. Tech. Conf. Spring, 2004.

[10] G. J. Foschini, and M. J. Gans, "On limits of wireless communication in a fading environment when using multiple antennas," Wireless Personal Commun., vol. 6, no. 3, pp. 311-355, Mar. 1998.

[11] K. K. Wong, and A. Paulraj, "Near maximum-likelihood detection with reduced-complexity for multiple-input single-output antenna systems," in Proc. Asilomar Conf. on Signals, Systems, and Computers, Nov. 2004. 


$$
\mathcal{C}(n, \mathrm{SNR})=4\left[1+\frac{2|\mathcal{Q}|^{n-1}-1}{\sqrt{2 \pi}} \cdot \frac{\mu}{\sqrt{n}} \cdot \frac{\sqrt{\mathrm{SNR}}}{\sqrt{\mathrm{SNR}+1}} \int_{-\frac{\pi}{2}}^{\frac{\pi}{2}} \cos ^{n} \theta e^{-\frac{\mu^{2}}{2 n}\left(\frac{\mathrm{SNR}}{\operatorname{SNR}+1}\right) \sin ^{2} \theta} d \theta\right]
$$

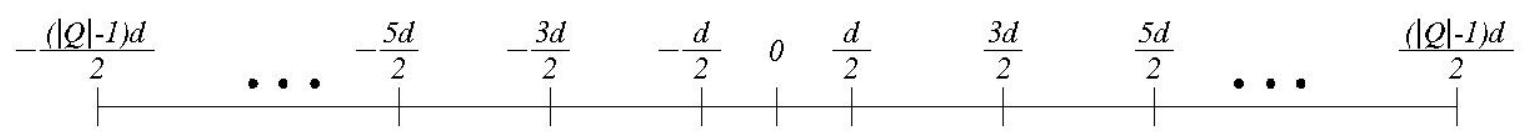

Fig. 2. Signal constellations for $|\mathcal{Q}|$-PAM.

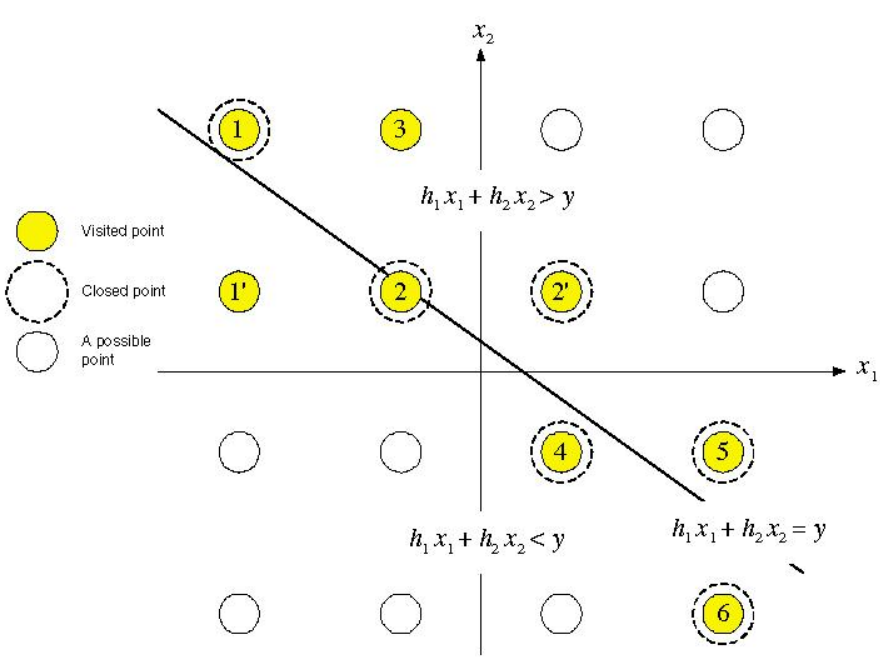

Fig. 1. A diagram showing the decoding sequence of planar detection when $\mathcal{Q}=\{-3,-1,1,3\}$ and $n=2$.

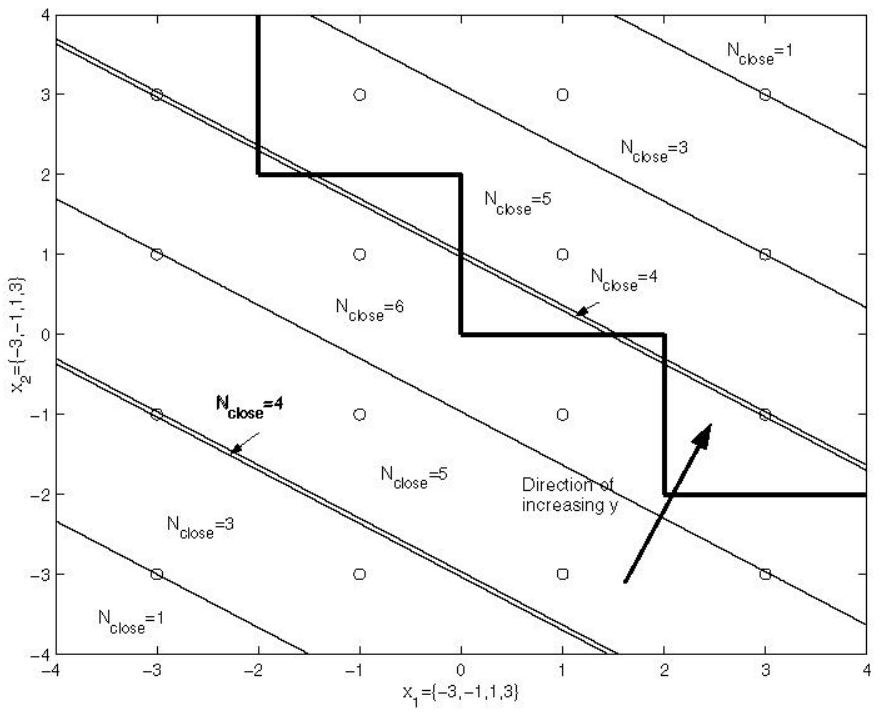

Fig. 3. An example showing how $\mathcal{N}_{\text {close }}$ depends on $y$ assuming the decoding line $2 x_{1}+3 x_{2}=y$ and $\mathcal{Q}=\{-3,-1,1,3\}$. The regions where the decoding line lies are labelled with the corresponding values of $\mathcal{N}_{\text {close }}$.

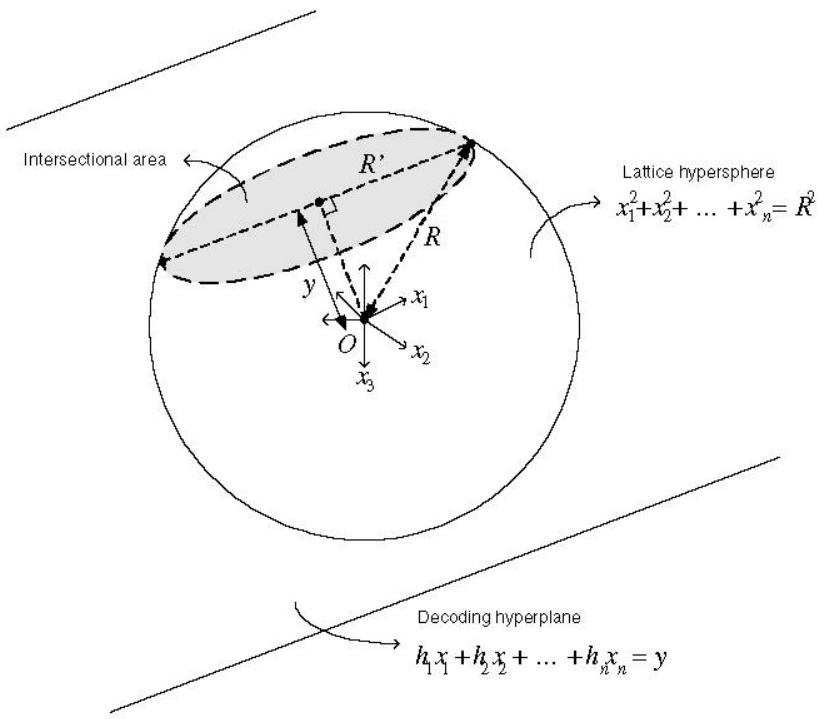

Fig. 4. Geometry of the decoding hyperplane and the lattice hypersphere.

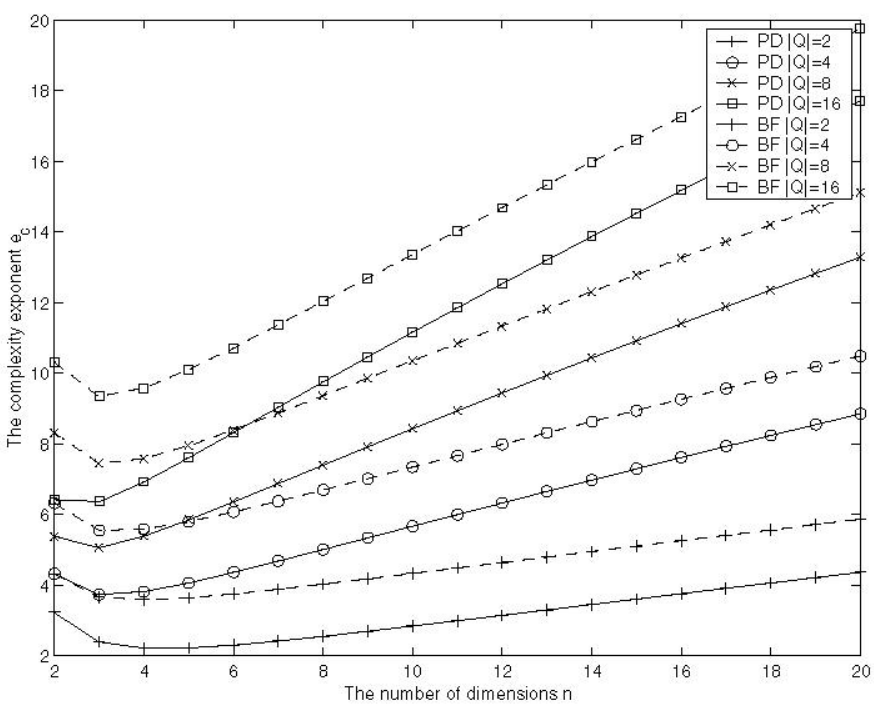

Fig. 5. The complexity exponent as a function of $n$ for various $|\mathcal{Q}|$ and $\mathrm{SNR}=15 \mathrm{~dB}$. PD refers to planar detection and $\mathrm{BF}$ refers to brute-force ML detection 